\title{
Modal analysis of Bragg onion resonators
}

\author{
Yong Xu, Wei Liang, and Amnon Yariv \\ Department of Applied Physics, MS 128-95, California Institute of Technology, Pasadena, California 91125
}

James G. Fleming and Shawn-Yu Lin

Sandia National Laboratories, Albuquerque, New Mexico 87185-1080

Received September 22, 2003

\begin{abstract}
From analysis of the high $Q$ modes in a Bragg onion resonator with an omnidirectional reflector cladding, we establish a close analogy between such a resonator and a spherical hollow cavity in perfect metal. We demonstrate that onion resonators are ideal for applications that require a large spontaneous-emission factor $\beta$, such as thresholdless lasers and single-photon devices. (C) 2004 Optical Society of America

OCIS codes: $230.5750,220.4000$.
\end{abstract}

Recently, much research has been devoted to the possibility of using optical microcavities, such as silica microspheres,${ }^{1}$ semiconductor micropillars,${ }^{2}$ and semiconductor microdisks, ${ }^{3}$ to significantly modify spontaneous-emission processes. In a previous Letter, ${ }^{4}$ we proposed to approximately realize a fully spherically symmetric Bragg resonator ${ }^{5-7}$ with an onionlike geometry. Such onion resonators, which consist of a spherical air core bound by Bragg cladding pairs composed of $\mathrm{SiO}_{2}$ and $\mathrm{Si}$, have been fabricated through a combination of etching and chemical-vapor deposition. A scanning electron microscope image of the onion resonator is shown in Fig. 1 . Since the photon confinement in the Bragg onion resonator is provided by Bragg reflection instead of total internal reflection, the onion resonator can combine a high quality factor, $Q>10^{6}$, with a cavity dimension of a few micrometers. ${ }^{4}$ This combination is difficult to achieve in other types of optical resonators such as silica microspheres, where optical confinement is provided by total internal reflection so that the losses increase with reduced radial size as a result of diminished confinement. ${ }^{1}$ Furthermore, because of the large index contrast between $\mathrm{SiO}_{2}$ and $\mathrm{Si}$, the spherical Bragg cladding forms an omnidirectional reflector, ${ }^{8}$ which reflects light incident from air irrespective of the incident angle and effectively isolates the onion core from the surrounding free space. In this Letter we analyze the modal spectrum of an onion resonator and establish a close analogy between the onion cavity and a hollow spherical cavity in perfect metal. We derive a simple formula that approximates the frequencies of the high $Q$ modes in a Bragg onion resonator well. From this formula, we derive several features that distinguish the high $Q$ modes in the onion resonator from the whispering-gallery modes in dielectric microspheres. Through an analysis of the spontaneous-emission processes in an onion resonator, we demonstrate that such resonators are particularly attractive for applications that require a large spontaneous-emission factor, such as thresholdless lasers $^{9}$ and single-photon devices. ${ }^{2,3}$

We begin the analysis by approximating the Bragg onion resonator with a spherically symmetric Bragg resonator. Because of the spherical symmetry, a resonator mode can be labeled by a pair of angular modal number $l$ and $m$, with $l \geq 1$ and $-l \leq m \leq l$. The frequencies of modes with the same $l$ but different $m$ are
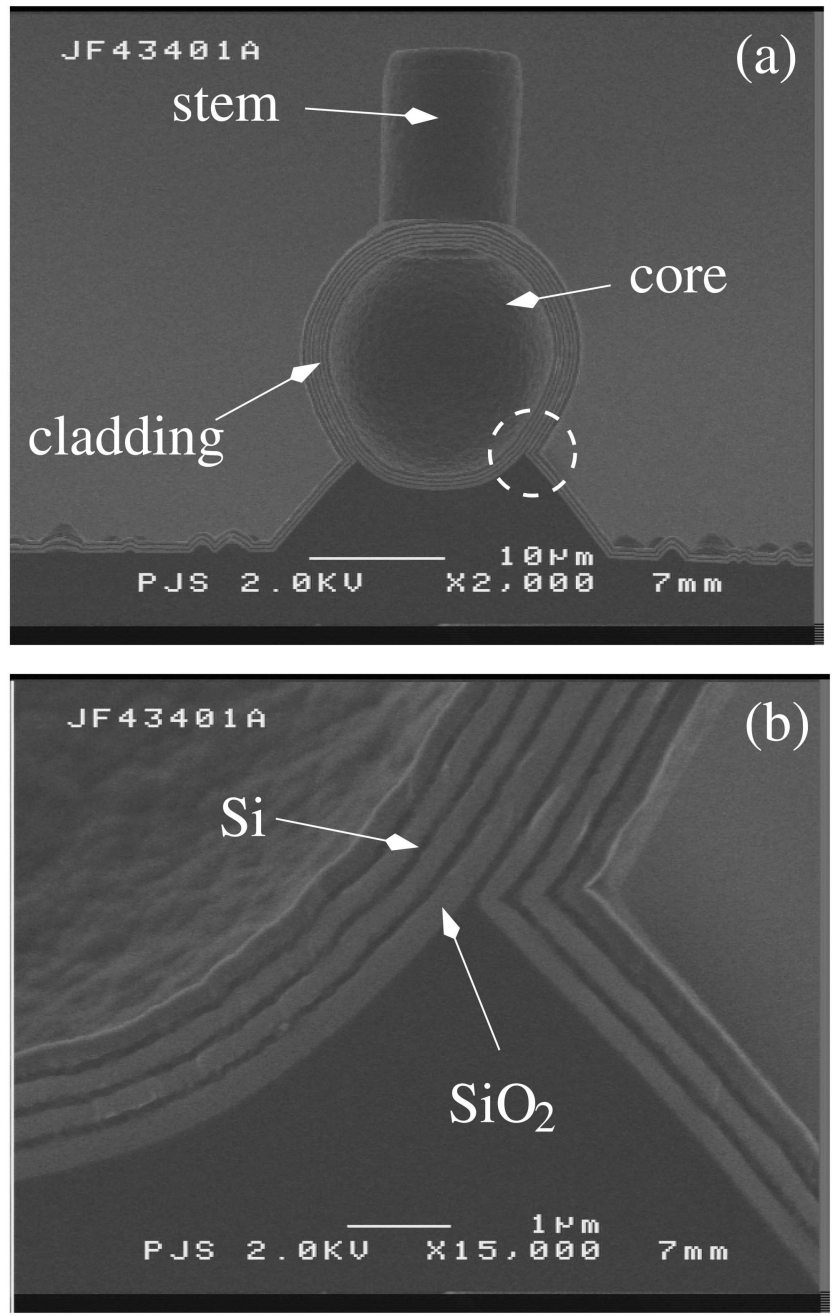

Fig. 1. Scanning electron microscope images of (a) an air-core Bragg onion resonator with $\mathrm{Si} / \mathrm{SiO}_{2}$ cladding pairs and (b) image of the onion resonator cladding, which corresponds to the region within the dashed circle in (a). 
identical. The resonator modes can be further classified as either transverse electric (TE) or transverse magnetic (TM). The frequencies and quality factors of the resonator modes can be calculated with the transfer matrix method developed in Ref. 4. In this Letter we choose to study a representative example and consider an air-core Bragg onion resonator with a radius of $r_{\mathrm{co}}=6.6 \mu \mathrm{m}$. The onion cladding is composed of eight pairs of $\mathrm{Si} / \mathrm{SiO}_{2}$ layers. The refractive index and thickness of the Si layers are $n_{\mathrm{Si}}=3.5$ and $L_{\mathrm{Si}}=0.111 \mu \mathrm{m}$, respectively, and the corresponding parameters for the $\mathrm{SiO}_{2}$ layers are $n_{\mathrm{SiO}_{2}}=1.5$ and $L_{\mathrm{SiO}_{2}}=0.258 \mu \mathrm{m}$. In labeling different onion modes, we keep the angular quantum number $l$ but drop $m$, because of the $2 l+1$-fold degeneracy. Furthermore, we introduce a radial quantum number $n$ and label the onion modes as $\mathrm{TE}_{l}{ }^{n}$ or $\mathrm{TM}_{l}{ }^{n}$, where we obtain $n$ by counting the radial wave-function nodes of the TE field that lies within the air core (we exclude the node in the proximity of the core-cladding interface). In Fig. 2(a) we show the radial dependence of the TE field of the $\mathrm{TE}_{3}{ }^{7}, \mathrm{TM}_{9}{ }^{5}$, and $\mathrm{TE}_{19}{ }^{1}$ modes. We notice that all three modes are very nearly zero at the onion core-cladding interface. This zero condition is the exact boundary condition for the electromagnetic field in a hollow spherical cavity bound by perfect metal and indicates a close analogy between the two seemingly different structures (one dielectric and one metallic). In Fig. 2(b) we compare the modal wavelength in these two types of cavities with the same core radius. For the metallic cavity, the modal frequency is given by ${ }^{10}$

$$
\begin{array}{r}
\left.\frac{\partial}{\partial r}\left[r j_{l}(k r)\right]\right|_{r=r_{\mathrm{co}}} \text { for TM modes, } \\
j_{l}\left(k r_{\mathrm{co}}\right)=0 \quad \text { for TE modes },
\end{array}
$$

where $k$ is the vacuum wave vector and $j_{l}(x)$ is the $l$ th-order spherical Bessel function. From Fig. 2(b), it is clear that the modes within the onion resonator bear a striking similarity to those in the hollow perfect metal cavity, with a maximum wavelength difference of less than $1 \%$. The similarity between a Bragg onion resonator and a hollow spherical cavity in perfect metal is not accidental, since the cladding parameters are chosen such that it forms an omnidirectional reflector within the wavelength range of Fig. 2(b) and behaves like a perfect metal. ${ }^{8}$ We notice that the simple relation given in Eq. (1) can be used to accurately describe the distribution of the eigenmode frequencies of a given onion resonator.

Unlike with whispering-gallery modes in dielectric microspheres, we observe that the frequencies of the onion modes in Fig. 2(b) can be further divided into distinctive groups according to an additional modal number $O_{l}{ }^{n}$, defined as

$$
O_{l}^{n}= \begin{cases}n+\frac{l-1}{2}+\delta_{l, 1} & \text { for TM modes } \\ n+\frac{l}{2} & \text { for TE modes }\end{cases}
$$

where $\delta_{l, 1}$ is 1 if $l=1$ and is 0 otherwise. Also, for small $l$ numbers, different modes with the same
$O_{l}{ }^{n}$ are nearly degenerate in frequency. This can be explained by solving Eq. (1) using the asymptotic form of the spherical Bessel function, which gives $k r_{\text {co }}=\left[n+(l-1) / 2+\delta_{l, 1}\right] \pi$, and consequently $\lambda_{\mathrm{TM}_{l}{ }^{n}}=2 r_{\mathrm{co}} / O_{l}{ }^{n}$ for the $\mathrm{TM}_{l}{ }^{n}$ mode. Similarly, in the asymptotic limit, we have $\lambda_{\mathrm{TE}_{l}}=2 r_{\mathrm{co}} / O_{l}{ }^{n}$ for the $\mathrm{TE}_{l}{ }^{n}$ mode. For the three groups of modes in Fig. 2(b) with $O_{l}{ }^{n}$ equal to 8, 8.5, and 9, this asymptotic formula $\lambda=2 r_{\mathrm{co}} / O_{l}{ }^{n}$ predicts the modal wavelength to be $1.650,1.553$, and $1.467 \mu \mathrm{m}$, respectively, in very good agreement with the exact results. The asymptotic approximation starts to break down as the angular quantum number $l$ becomes larger. However, even in this case, the high $Q$ modes still fall into distinctive groups, each characterized by a unique $O_{l}{ }^{n}$.

In Ref. 4 we demonstrated that onion resonator modes, even with relatively small $l$, can have quality factors exceeding $10^{6}$. This feature also applies to the onion resonator considered in this Letter and is quite different from that of dielectric microspheres, where modes with small $l$ in general have very low quality factors. For example, the quality factors of
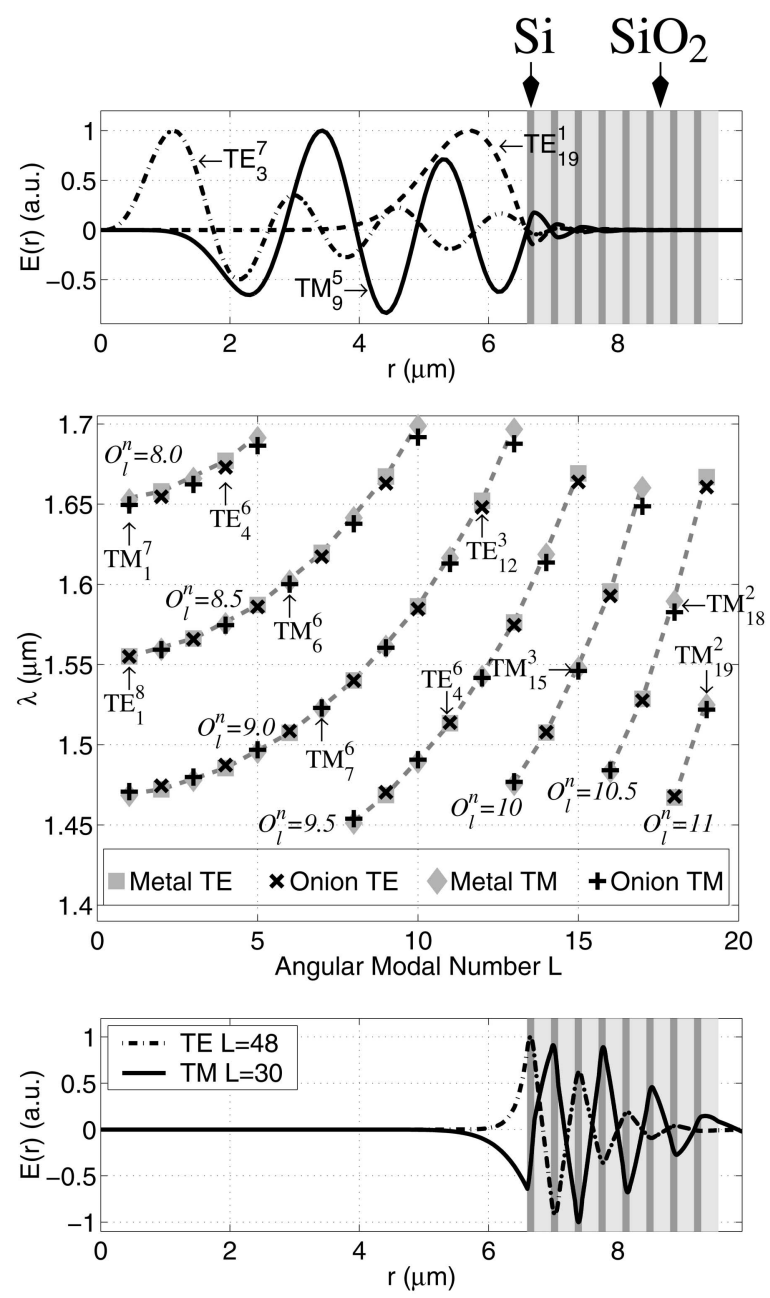

(c)

Fig. 2. (a) TE field of several high $Q$ modes in an onion cavity. (b) The resonance wavelength of the onion modes as a function of the angular modal number $l$, together with the wavelength of resonant modes in a hollow spherical cavity in perfect metal. (c) Cladding modes of the onion structure. 
the $\mathrm{TE}_{3}{ }^{7}$ and $\mathrm{TM}_{9}{ }^{5}$ modes shown in Fig. 2(a) are $1.07 \times 10^{7}$ and $7.21 \times 10^{6}$, respectively. Fundamentally, the difference between dielectric microspheres and onion cavities is a result of different confinement mechanisms, i.e., total internal reflection versus Bragg reflection. For both structures, we can identify the mode with angular modal number $l$ as a light ray striking the core-cladding interface with a transverse wave vector $k_{t}=l / r_{\mathrm{co}}$. For a microsphere with refractive index $n$, the total internal reflection requires $2 \pi n / \lambda \geq k_{t} \geq 2 \pi / \lambda$, which consequently gives the range of the angular momentum $l$ as $2 \pi n r_{\mathrm{co}} / \lambda \geq l \geq 2 \pi r_{\mathrm{co}} / \lambda$. On the other hand, for the onion resonator with an omnidirectional reflection cladding, any light ray that is incident at the core-cladding interface, as long as it satisfies $k_{t} \leq 2 \pi / \lambda$, will be reflected irrespective of the incidence angle. Therefore the onion mode $l$ should satisfy $2 \pi r_{\mathrm{co}} / \lambda \geq l \geq 1$. Outside this range, optical modes still exist in onion structures, but they should be regarded as the cladding modes, since their electromagnetic fields are concentrated within the cladding region. Two such cladding modes are shown in Fig. 2(c). Additionally, since the omnidirectional onion cladding can reflect light that is incident at any angle, we expect that the air-core radius can be reduced to $1-2 \mu \mathrm{m}$ while high quality factors at the level of $10^{6}-10^{7}$ are maintained. This feature is of great importance for cavity QED applications and cannot be achieved in silica microspheres in which the cavity $Q$ factors exponentially decrease as we reduce the microsphere radius. ${ }^{1}$

An important application envisaged for Bragg onion resonators is for optical devices that requires a large spontaneous-emission factor. In any high $Q$ cavity, we can separate the total spontaneous-emission rate $\gamma_{\text {tot }}$ into a resonant part $\gamma_{\text {res }}$, defined as the emission rate into a given high $Q$ mode, and a background part $\gamma_{\mathrm{bg}}=\gamma_{\mathrm{tot}}-\gamma_{\mathrm{res}}$, which accounts for spontaneous emission into the rest of the electromagnetic modes. For microcavities such as microspheres or micropillars, the background emission rate $\gamma_{\mathrm{bg}}$ is essentially the bulk spontaneous-emission rate $\gamma_{\text {bulk }}$, whereas the resonant emission rate $\gamma_{\text {res }}$ can be estimated as ${ }^{11}$

$$
\frac{\gamma_{\mathrm{res}}}{\gamma_{\mathrm{bulk}}} \approx \frac{1}{4 \pi^{2}} \frac{\lambda^{3}}{V} \frac{1}{\frac{1}{Q}+\frac{\Gamma}{\omega}},
$$

where $V$ is the modal volume, $\omega$ and $Q$ are the frequency and the quality factor of the cavity mode, respectively, and $\Gamma$ is the linewidth of the light-emitting material. Therefore, to achieve a large spontaneousemission factor $\beta$, which is $\gamma_{\mathrm{res}} /\left(\gamma_{\mathrm{res}}+\gamma_{\mathrm{bg}}\right)$, the resonant emission rate $\gamma_{\text {res }}$ needs to be enhanced to a value much larger than $\gamma_{\text {bulk }}$. According to Eq. (3), this requires a reduction of the cavity volume, an increase of the cavity $Q$, and a choice of a light emitter with narrow linewidth $\Gamma$. In practice, the large spectral width of common light emitters (for example, the gain bandwidth is of the order of $20 \mathrm{~nm}$ in erbium-doped glass ${ }^{12}$ ) has turned out to be a major obstacle.
We can achieve a large spontaneous-emission factor in a Bragg onion resonator through a fundamentally different approach. We notice that the omnidirectional cladding can result in almost complete isolation of the onion core from free space. For light emitters in the core region, there are only two remaining background spontaneous decaying channels: (1) coupling to the onion cladding modes and (2) coupling to the free space through the onion stem, as shown in Fig. 1. The coupling to the onion cladding modes can be ignored, since (i) only a very small amount of the cladding field exists within the core region, as can be seen from Fig. 2(c), and (ii) the cladding mode coupling can be further reduced by designing the onion structures such that the light-emission frequency and the cladding mode frequencies are spectrally well separated. This means the background emission rate will be dominated by the stem coupling, which can be estimated from the fraction of the solid angle spanned by the stem. Assuming the stem radius to be $r_{\text {stem }}$, we have $\gamma_{\mathrm{bg}}=\gamma_{\mathrm{bulk}}\left(r_{\mathrm{stem}} / 2 r_{\mathrm{co}}\right)^{2}$. If we take the stem radius as $1 \mu \mathrm{m}$ (a value within the reach of actual fabrication) and the core radius as $6.6 \mu \mathrm{m}$, the background spontaneous-emission rate is only $0.6 \%$ of the bulk value. Thus we conclude that, in a Bragg onion resonator, it is possible to inhibit background spontaneous emission by at least 2 orders of magnitude. As a result, even for $\gamma_{\text {res }} \sim \gamma_{\text {bulk }}$, we can obtain a spontaneous-emission factor of $\sim 99 \%$, which allows us to use a much wider range of light-emitting materials, such as erbium-doped glass and organic dyes, and may even lead to room-temperature operation.

Y. Xu's e-mail address is yong@its.caltech.edu.

\section{References}

1. J. R. Buck and H. J. Kimble, Phys. Rev. A 67, 033806 (2003).

2. J. M. Gérard, B. Sermage, B. Gayral, B. Legrand, E. Costard, and V. Thierry-Mieg, Phys. Rev. Lett. 81, 1110 (1998).

3. A. Kiraz, P. Michler, C. Becher, B. Garyral, A. Imamoğlu, L. Zhang, E. Hu, W. V. Schoenfeld, and P. M. Petroff, Appl. Phys. Lett. 78, 3932 (2001).

4. Y. Xu, W. Liang, A. Yariv, J. G. Fleming, and S. Lin, Opt. Lett. 28, 2144 (2003).

5. D. Brady, G. Papen, and J. E. Sipe, J. Opt. Soc. Am. B 10, 644 (1993).

6. M. A. Kaliteevski, S. Brand, R. A. Abram, and V. V. Nikolaev, J. Mod. Opt. 48, 1503 (2001).

7. D. D. Smith and K. A. Fuller, J. Opt. Soc. Am. B 19, 2449 (2002).

8. J. N. Winn, Y. Fink, S. Fan, and J. D. Joannopoulos, Opt. Lett. 23, 1573 (1998).

9. Y. Yamamoto, S. Machida, and G. Björk, Phys. Rev. A 44, 657 (1991).

10. J. D. Jackson, Classical Electrodynamics, 3rd ed. (Wiley, New York, 1999).

11. M. P. van Exter, G. Niehuis, and J. P. Woerdman, Phys. Rev. A 54, 3553 (1996).

12. E. Desurvire, D. Bayart, B. Desthieux, and S. Bigo, Erbium-Doped Fiber Amplifiers, Device and System Developments (Wiley, New York, 2002). 